This item was submitted to Loughborough's Research Repository by the author.

Items in Figshare are protected by copyright, with all rights reserved, unless otherwise indicated.

\title{
The inflammatory response to a wheelchair half-marathon in people with a spinal cord injury - the role of autonomic function
}

\section{PLEASE CITE THE PUBLISHED VERSION}

https://doi.org/10.1080/02640414.2019.1586296

\section{PUBLISHER}

(C) Taylor \& Francis

\section{VERSION}

AM (Accepted Manuscript)

\section{PUBLISHER STATEMENT}

This is an Accepted Manuscript of an article published by Taylor \& Francis in Journal of Sports Sciences on 5 March 2019, available online: http://www.tandfonline.com/10.1080/02640414.2019.1586296.

\section{LICENCE}

CC BY-NC-ND 4.0

\section{REPOSITORY RECORD}

Hoekstra, Sven, Christof Leicht, Yoshi-Ichiro Kamijo, Tokio Kinoshita, Ben T. Stephenson, Vicky GooseyTolfrey, Nicolette Bishop, and Fumihiro Tajima. 2019. "The Inflammatory Response to a Wheelchair Halfmarathon in People with a Spinal Cord Injury - the Role of Autonomic Function". figshare. https://hdl.handle.net/2134/37189. 


\section{The inflammatory response to a wheelchair half-marathon in people with a spinal cord injury - the role of autonomic function}

1. The Peter Harrison Centre for Disability Sport; School of Sport, Exercise, and Health Sciences; Loughborough University; Loughborough; UK

2. Department of Rehabilitation Medicine; Wakayama Medical University; Wakayama; Japan

14 The authors declare no conflicts of interest.

15 interleukin-6; heat shock protein 72; wheelchair racing; upper-body exercise; sympathetic

16 response; adrenaline

17 Word count: 4283

18 Email addresses in the order of author list:

19 s.p.hoekstra@lboro.ac.uk; c.a.leicht@lboro.ac.uk; yoshikmj@gmail.com; 20 ycksg874@yahoo.co.jp; b.t.stephenson@ lboro.ac.uk; v.l.tolfrey@ lboro.ac.uk; 21 n.c.bishop@lboro.ac.uk; tajibun@gmail.com

22

23

24

\section{Corresponding author:}

26 Christof A. Leicht

27 The Peter Harrison Centre for Disability Sport; School of Sport, Exercise and Health

28 Sciences; Loughborough University

29 Loughborough LE11 3TU, UK

30 c.a.leicht@lboro.ac.uk 


\section{Abstract}

33 This study investigates the relationship between autonomic function and the inflammatory 34 response to a wheelchair half-marathon in people with a spinal cord injury (SCI). Seventeen 35 wheelchair athletes with a cervical SCI (CSCI, N=7) and without CSCI (NON-CSCI, N=10) 36 participated in a wheelchair half-marathon. Blood was taken prior, post and $1 \mathrm{~h}$ post-race to 37 determine the concentrations of adrenaline, noradrenaline, extracellular heat shock protein 72 38 (eHsp72) and interleukin-6 (IL-6). A sit-up tilt test was performed to assess autonomic 39 function at rest. CSCI showed a lower supine ratio of the low and high frequency power of 40 the variability in RR intervals (LF/HF RRI, $p=0.038$ ), total and low frequency power of the 41 systolic blood pressure variability (TP SBP, $p<0.001$; LF SBP, $p=0.005$ ) compared to NON42 CSCI. Following the race, catecholamine concentrations increased only in NON-CSCI 43 ( $p$ 0.036). The increase in IL-6 post-race was larger in NON-CSCI $(p=0.040)$. Post-race 44 catecholamine levels explained $60 \%$ of the variance in the IL-6 response $(r=0.77, p=0.040)$, 45 which was further increased when the resting autonomic function indices were added to the 46 regression model $\left(\mathrm{R}^{2}>81 \%, p<0.012\right)$. In summary, the dampened acute inflammatory 47 response to a wheelchair half-marathon in CSCI was strongly associated with the autonomic 48 dysfunction present in this group. 


\section{Introduction}

55 Chronic low-grade inflammation, characterised by elevated resting levels of pro-

56 inflammatory proteins (e.g. interleukin-6 (IL-6) and extracellular heat shock protein 72

57 (eHsp72)) and strongly associated with chronic diseases such as type 2 diabetes mellitus and 58 cardiovascular disease, is more prevalent in people with a spinal cord injury (SCI) compared 59 to members of the able-bodied population (Bauman \& Spungen, 2008). This might be the 60 consequence of a physically inactive lifestyle and the physiological changes accompanying 61 the injury (Martin Ginis et al., 2010). Exercise training is widely recognised as a means to 62 combat chronic low-grade inflammation (Petersen \& Pedersen, 2005), partly as a result of the 63 acute inflammatory response induced by each bout. Although transiently elevating pro64 inflammatory proteins such as IL-6, the subsequent longer lasting anti-inflammatory response 65 can lower resting levels of these proteins when engaging in exercise on a regular basis 66 (Petersen \& Pedersen, 2005). During and shortly after exercise, the primary source of 67 circulating IL-6 concentrations is skeletal muscle (Steensberg et al., 2000). Despite a smaller 68 active muscle mass involved, upper-body exercise has been shown effective in the induction 69 of an acute inflammatory response (Sasaki et al., 2014; Umemoto et al., 2011). However, this 70 response might be attenuated in people with a cervical SCI (CSCI) (Paulson et al., 2013).

71 Persons with CSCI have a smaller muscle mass and impaired autonomic function 72 compared to able-bodied individuals and people with paraplegia, both impacting on their 73 exercise capacity (West et al., 2015). There is limited research into the influence of these 74 factors on the acute inflammatory response to exercise. Paulson et al. (2013) reported an 75 attenuated IL-6 response to a strenuous bout of exercise in CSCI compared to SCI; a finding 76 that was replicated in a wheelchair racing setting (Ogawa et al., 2014). Together with the 77 attenuated IL-6 response, both studies reported a blunted adrenaline and noradrenaline 
response to the exercise bout in the CSCI group (Ogawa et al., 2014; Paulson et al., 2013),

79 suggesting a major role of catecholamines in the dampened acute inflammatory response to 80 exercise present in people with CSCI. Indeed, adrenaline infusion in resting able-bodied

81 individuals results in increased plasma IL-6 concentrations (Sondergaard et al., 2000;

82 Steensberg et al., 2001), potentially via the increase of intracellular cyclic adenosine

83 monophosphate (cAMP) following the stimulation of $\beta$-receptors on skeletal muscle with 84 adrenaline (Sondergaard et al., 2000).

Apart from resulting in lower resting and exercise-induced plasma catecholamine 86 concentrations, CSCI can lead to the disruption of sympathetic innervation of the heart, as members of this population have an impaired blood pressure regulation and heart rate (HR) attained during maximal exercise does often not exceed $~ 130$ bpm (West et al., 2015). Therefore, although an attenuated catecholamine response to exercise is a characteristic of autonomic dysfunction, the latter affects a plethora of other physiological processes that may influence the acute inflammatory response to exercise. As autonomic completeness of a spinal injury varies between people as a result of the potential sparing of autonomic fibres below the lesion (Krassioukov, 2009), the impact of autonomic dysfunction on the inflammatory response to exercise may also differ between people with SCI.

For a more comprehensive assessment of autonomic function than using exerciseinduced plasma catecholamine concentrations alone (Paulson et al., 2013; Ogawa et al., 2014), additional tests exist (Krassioukov, 2009). The sit-up tilt test, for instance, can be used to detect abnormal changes in HR and blood pressure in response to a passive tilt manoeuvre (Claydon \& Krassioukov, 2008). Moreover, Claydon \& Krassioukov (2008) reported that frequency blood pressure and heart rate variability measures taken in a supine position are 
predictive of a range of clinical measures of autonomic dysfunction, such as for instance orthostatic hypotension.

The present study therefore extends on previous research (Ogawa et al., 2014;

Paulson et al., 2013) by incorporating autonomic function indices measured at rest in addition to the assessment of the catecholamine response following exercise to investigate the association of autonomic function with the inflammatory response to a wheelchair halfmarathon. In addition, this study investigates the potential of upper-body exercise in SCI to acutely elevate eHsp72 concentrations, a relatively novel marker implicated in chronic lowgrade inflammation (Johnson \& Fleshner, 2006). Together, this can provide further insight into factors that influence the acute inflammatory response to exercise and inform strategies to reduce chronic low-grade inflammation in people with CSCI.

\section{Methods}

Participants were seventeen male recreational wheelchair athletes with $\mathrm{CSCI}(\mathrm{N}=7)$ or without CSCI (NON-CSCI, N=10), the latter group including individuals with a spinal lesion below the cervical level $(\mathrm{N}=6)$, spina bifida $(\mathrm{N}=2)$, polio $(\mathrm{N}=1)$ and myotonia congenita $(\mathrm{N}=1)$ (Table 1). Participants took part in the wheelchair half-marathon of Oita 2016. The study was approved by the local ethics committees of Loughborough University (United Kingdom) and Wakayama University (Japan) and participants gave informed consent prior to participation.

Sit-up tilt test

125 for the assessment of autonomic function. All tests were performed using the same 
wheelchair with adjustable back rest and in a room set at $25^{\circ} \mathrm{C}$. Participants were rested for 5 minutes in a supine position and were then elevated into the sitting position, which they maintained for another 5 minutes. Participants were instructed to breathe at a frequency of $0.25 \mathrm{~Hz}$. Blood pressure was measured beat-by-beat at the wrist (MUB101-50, MediSense Inc., Tokyo, Japan) whilst a brachial blood pressure cuff (STBP-780, Colin, Komaki, Japan) was used for the calibration. Both cuffs were situated at the level of the heart for the full duration of the test. Heart rate was continuously monitored using a 7-lead electrocardiogram (PhysioFlow Lab-1, Manatec Biomedical, Paris, France). Following the test, participants reported their height, body mass, wheelchair racing experience and average time spent training.

To obtain HR and blood pressure variability measures in the frequency domain, beatto-beat mean arterial pressure and HR were obtained by integrating the respective signals within each cardiac cycle. Cardiac cycles were determined based on the diastolic intervals of the beat-to-beat blood pressure signal. Beat-to-beat mean arterial pressure and HR were first linearly interpolated and resampled at $2 \mathrm{~Hz}$ and then detrended by subtracting their third polynomial. Subsequently, the beat-to-beat time series were used for the spectral analyses based on the Welch algorithm. Each time series was subdivided into successive 256-point Hann windows that overlapped by $50 \%$ before fast Fourier transform analysis (Van der Scheer et al., 2018). Outcome measures used to reflect autonomic function were: the largest drop in blood pressure following the onset of the sitting position (to detect orthostatic hypotension $(\mathrm{OH})$, defined as a drop in $\mathrm{SBP}>20 \mathrm{mmHg}$ or $\mathrm{DBP}>10 \mathrm{mmHg}$ (Freeman et al., 2011 ), the ratio of the power in the low (power $=0.07-0.20 \mathrm{~Hz}$ ) and high frequency (power $=0.20-35 \mathrm{~Hz})$ domain of the heart rate variability $(\mathrm{LF} / \mathrm{HF} \mathrm{RRI})$, total power and power in the low frequency domain of systolic blood pressure variability (TP SBP and LF SBP, respectively) in the supine position (Claydon \& Krassioukov, 2008). These variability 
measures were chosen as they have been shown reliable in people with SCI (Ditor et al., 2005)

and to strongly correlate with clinical symptoms of autonomic dysfunction (Claydon \& Krassioukov, 2008). Additionally, the root mean square differences of successive R-R intervals in the supine position (RMSSD) was included as a time domain autonomic function index.

\section{Wheelchair half-marathon}

The Oita wheelchair half-marathon is a $21.1 \mathrm{~km}$ race on relatively flat terrain in the city centre. The race started at $10 \mathrm{am}$ in relatively mild conditions $\left(23^{\circ} \mathrm{C}, 56 \%\right.$ relative humidity). Participants had refrained from exercise in the 24 hours prior to the race. While food and liquid ingestion were not controlled due to the potential interference with the race practices of the athletes, these were reported using a food diary. All participants reported to have consumed a carbohydrate-rich breakfast in the morning of the race and to have consumed a sports drink during the race. Heart rate was continuously measured using a Garmin monitor (Garmin Edge 500, US). Blood was drawn from an antecubital vein prior to the warm-up for the race (pre), directly after finishing the race (post) and $1 \mathrm{~h}$ post-race $(1 \mathrm{~h}$ post) into a glass serum separation and a $\mathrm{K}_{3}$ EDTA tube of which respectively serum and plasma were extracted and stored at $-80{ }^{\circ} \mathrm{C}$ until analysis. These time-points were chosen based on previous studies on the acute inflammatory response to endurance-type exercise in able-bodied (Fehrenbach et al., 2005; Fischer, 2006) and individuals with disability (Ogawa et al., 2014). Interleukin-6 (High sensitivity; R\&D systems, UK) and eHsp72 (Amp`d high sensitivity; Enzo life sciences, US) were analysed in serum using a quantitative sandwichtype enzyme-linked immunosorbent assay (CV: $8.2 \%$ and 6.3\%, respectively), while adrenaline and noradrenaline were analysed in plasma by high-performance liquid 
chromatography. Haemoglobin and haematocrit were determined using an automated cell counter (MEK-6400, Nihon Koden, Tokyo, Japan) and were used to correct the outcome markers for changes in plasma volume resulting from the race (Dill and Costil, 1964).

\section{Statistical analyses}

The participants were divided into two groups based on lesion level (regardless of completeness), resulting in a CSCI and NON-CSCI group. All data were checked for normality with the Shapiro-Wilk test, after which the data were log-transformed when this assumption was violated. Data were checked for sphericity using Levene`s test. Comparisons of athlete characteristics and responses to the sit-up tilt test between CSCI and NON-CSCI were assessed using independent student T-tests. Two-way repeated measures ANOVAs were used to assess changes in IL-6, eHsp72, adrenaline and noradrenaline in CSCI and NON-CSCI following the race. $95 \%$ confidence intervals of the difference in participants characteristics between both groups were computed, while effect sizes (ES) Cohen`s d were calculated where appropriate, whereby an ES of $0.20,0.5$ and 0.80 refers to a small, moderate or large effect, respectively (Cohen, 1992). The ES for a time x group interaction was calculated by comparing the pre-post change scores in each group. Using GPower 3.1.9.2, we calculated we would need six participants in both groups to detect a difference in the acute IL-6 response to exercise between the groups, with 90\% power, an $\alpha$ of 5\% and an effect size of 2.0 based on data reported by Ogawa et al. (2014).

To further assess the relationship of catecholamines and the autonomic function indices with the inflammatory response following the race, simple and multiple linear regression analysis using the whole sample was performed. Adrenaline and noradrenaline were entered into the model both together and individually and the $\mathrm{R}^{2}$ was used to describe 
the explained variance of the dependent variable. The same was done for LF/HF RRI,

RMSSD, LF SBP and TP SBP. Finally, adrenaline and noradrenaline together with a heart rate and blood pressure variability measure were entered into the regression model and the change in $\mathrm{R}^{2}$ as compared to the model with the catecholamines or sit-up tilt test indices only was tested using ANOVA. Collinearity among the predictor variables was tested using

Tolerance and the Condition Index, whereby values higher than 1 and 15 respectively were considered concerning (Midi et al., 2010). All analyses were performed using the $23^{\text {rd }}$ version of SPSS and statistical significance was defined as $p<0.05$.

\section{Results}

Sit-up tilt test

Five participants demonstrated $\mathrm{OH}$ in response to the tilt manoeuvre, four of which were in the CSCI group and one in NON-CSCI. Athletes in the CSCI group showed a larger drop in SBP in response to the tilt manoeuvre compared to NON-CSCI ( $p=0.017$; ES: 1.36 ). LF/HF RRI ( $p=0.038 ; \mathrm{ES}: 1.30), \operatorname{LF~SBP}(p=0.005 ; \mathrm{ES}: 1.86)$ and TP SBP $(p<0.001 ; \mathrm{ES}$ : 2.37) differed significantly between the two groups, with NON-CSCI showing larger values.

The RMSSD did not differ between both groups $(p=0.87)$ (Table 1). As such, the remaining of this manuscript focusses on the frequency rather than time domain autonomic function indices at rest.

*****Table 1 near here******

Wheelchair half-marathon

At rest, CSCI had lower levels of adrenaline ( $p=0.003$; ES: 1.62$)$ and noradrenaline ( $p<0.001$; ES: 3.20$)$ compared to NON-CSCI. While there was no difference in baseline IL-6 
22

serum concentrations $(p=0.519)$, a strong trend for higher resting eHsp72 concentrations for

CSCI compared to NON-CSCI was present ( $p=0.055$; ES: 1.04) (Fig. 1).

The NON-CSCI group took significantly less time to complete the race than the CSCI athletes $(1.06(0.21)$ versus $1.43(0.38) \mathrm{h}, p=0.026$; ES: 1.21$)$. The average HR during the race was significantly higher for NON-SCI compared to CSCI (145 (32) versus 103 (20) bpm, $p=0.029 ; \mathrm{ES}: 1.43)$. There was a trend for a higher peak HR in the NON-CSCI group (166 (41) versus 129 (18) bpm, $p=0.087$; ES: 1.04).

Following the race, plasma adrenaline and noradrenaline concentrations were increased in NON-CSCI $(p<0.036$; ES: 1.60 and 1.58 for adrenaline and noradrenaline, respectively), but not in CSCI ( $p>0.113$; ES: 0.63 and 0.84 for adrenaline and noradrenaline, respectively). The increase in serum IL-6 concentrations in response to the race was larger in NON-CSCI compared to CSCI ( $p=0.040$; ES: 0.71), although the latter group showed a significant increase in IL-6 as well ( $p=0.033$; ES: 1.03). Extracellular heat shock protein 72 did not increase in either of the groups (NON-CSCI: $p=0.338$ and CSCI: $p=0.116$ ) (Fig. 1). Since both IL-6 and eHsp72 concentrations did not change from post to $1 \mathrm{~h}$ post $(p>0.391)$, further analyses were performed using the serum concentrations of both markers immediately following the race only.

****** Figure 1 near here******

Association of autonomic function with the inflammatory response

The relationship between the autonomic function indices, catecholamines and the inflammatory response to the race is illustrated in Table 2 and Figure 2. Post-race levels of 


\section{3}

adrenaline and noradrenaline combined explained $60 \%$ of the variance in the IL-6 concentrations following to the race $(p=0.04)$, while adrenaline alone explained $44 \%$ of the variance ( $p=0.007)$. The LF/HF RRI and TP SBP both individually explained a significant proportion of the variance in post-race plasma adrenaline concentrations (29\% and 33\%, respectively; $p<0.042$ ). The model including combination of LF/HF RRI with LF SBP or TP SBP did not significantly explain the adrenaline response $(p>0.08)$. The variance in the IL-6 response could not be explained by one of the autonomic function indices in isolation (LF/HF RRI: $\mathrm{R}^{2}=8 \%, p=0.37$ RMSSD: $\mathrm{R}^{2}=11 \%, p=0.28$;F SBP: $\mathrm{R}^{2}=12 \%, p=0.27$;P SBP: $\mathrm{R}^{2}$ $=29 \%, p=0.07)$. The same was true for the combination of LF/HF RRI together with either of the two variability measures of systolic blood pressure $(p>0.281)$. The regression model including adrenaline, noradrenaline, LF/HF RRI and one of the systolic blood pressure variability measures (i.e. LF SBP or TP SBP) increased the explained variance of post-race IL-6 concentrations in comparison to the model including catecholamines only $\left(\mathrm{R}^{2}>81 \%\right.$, $p<0.012)$. The change in $\mathrm{R}^{2}$ compared with the model including catecholamines only was significant (adrenaline, noradrenaline, LF/HF RRI, TP SBP; $p=0.036$ ), or showed a trend towards significance (adrenaline, noradrenaline, LF/HF RRI, LF SBP; $p=0.07$ ). Of note, Tolerance and the Condition Index were $<0.54$ and $<8.87$, respectively, indicating no substantial collinearity among the predictor variables. Finally, athletes that presented with OH during the sit-up tilt test showed lower levels of adrenaline (104 (131) mmol/L versus 265 (243) mmol/L) and IL-6 (4.34 (1.08) pg/ml versus $10.21(6.84) \mathrm{pg} / \mathrm{ml})$ post-race, although only the difference in IL-6 reached statistical significance ( $p=0.192$; ES: 0.66 and $p=0.026$; ES: 0.96, respectively). 
275 Discussion

This study showed that the acute IL-6 response to a wheelchair half-marathon is attenuated in CSCI compared to NON-CSCI athletes, predominantly associated with the blunted catecholamine response observed in this group. Although autonomic function indices assessed at rest do not seem to be strong independent predictors for the IL-6 response to exercise, when used in combination with catecholamines they enhanced the predictive value of autonomic function assessments. This suggests that the influence of autonomic dysfunction on the dampened IL-6 response in people with CSCI is mediated by more than catecholamines only. Finally, eHsp72 was not elevated after the wheelchair half-marathon, suggesting that more intense or longer duration upper-body exercise is needed to increase its release into the circulation.

The resting autonomic function indices in the frequency domain (i.e. LF/HF RRI, LF SBP and TP SBP) differed between CSCI and NON-CSCI, indicating a difference in autonomic function between the two groups. Importantly, Claydon and Krassioukov (2008) observed a strong correlation between those measures and clinical outcomes of autonomic dysfunction (e.g. the catecholamine, HR and blood pressure response to the tilt manoeuvre as well as the sympathetic skin responses). As suggested by these authors, this may provide practitioners with an easy-to-use and non-invasive tool to assess autonomic function in people with SCI (Claydon \& Krassioukov, 2008). Further indicating a difference in autonomic function between NON-CSCI and CSCI, the latter showed lower plasma concentrations of adrenaline and noradrenaline at rest and in response to exercise.

Previous research using only catecholamines as an indication of autonomic function has highlighted the importance of an intact sympathetic nervous system for the elevation of IL-6 following exercise (Ogawa et al., 2014; Paulson et al., 2013). Indeed, in the current 
study catecholamines explained $60 \%$ of the variance in serum IL-6 levels post-race. Studies infusing adrenaline in persons at rest support the notion that adrenaline can independently elevate circulating IL-6 concentrations (Sondergaard et al. 2000; Steensberg et al. 2001). Animal studies using adrenergic receptor antagonists suggest that adrenaline stimulates IL-6 production by the activation of $\beta$-receptors (De Rijk et al. 1994), which in turn leads to an increase in intracellular cAMP (Langfort et al., 2003). This can directly stimulate IL-6 production (Zhang et al., 1988). Additionally, the impact of adrenaline on muscle glycogen breakdown may mediate its stimulating effect on IL-6 production (Jensen et al., 1999).

Indeed, exercise in a glycogen depleted state leads to an exacerbated acute IL-6 response (Bishop et al., 2001). However, the infusion of similar adrenaline levels as observed during exercise results in a much lower elevation in IL-6 when compared to exercise (Steensberg et al., 2001), suggesting that exercise provides additional stressors that cause the elevation of circulating IL-6 concentrations.

As circulating catecholamines seem to only partly explain the acute IL-6 response to exercise (Steensberg et al., 2001), other consequences of autonomic dysfunction such as the altered vascular tone and sympathetic innervation of the heart might further impact on the capacity to induce an inflammatory response through exercise (Paulson et al., 2013).

Autonomic function indices taken at rest are associated with clinical symptoms of autonomic dysfunction (Claydon \& Krassioukov, 2008), but also exercise performance (West et al., 2015). In the present study, the predictive value of the individual autonomic function indices measured at rest seems to be limited with regards to the inflammatory response. However, the addition of these measures to the regression model with catecholamines significantly enhanced the explained variance of post-race serum IL-6 concentrations. This suggests that a combination of exercise-induced (catecholamines) and resting autonomic function indices (supine LF/HF RRI, LF SBP and TP SBP) can provide additional insight into the role of 
sympathetic dysfunction in the acute IL-6 response to exercise in people with SCI. This may be used to inform individual exercise prescription as well as the creation of additional strategies to promote health in people with SCI (Leicht et al., 2015). From a mechanistic perspective, the added predictive value of autonomic function indices at rest suggests that the impact of autonomic dysfunction on the dampened acute inflammatory response to exercise is indeed mediated by other factors than blunted circulating catecholamine concentrations only. The close link between autonomic function and the catecholamine response to exercise makes it difficult to suggest what is accounted for by the resting autonomic function indices that is not accounted for by post-race catecholamine concentrations. In this respect it is noteworthy that athletes with autonomic complete CSCI might still experience autonomic reflexes below the lesion, resulting in spill-over of noradrenaline into the circulation (Leicht et al., 2013). Interestingly, one participant with CSCI showed a 4-fold and 2.5-fold increase in adrenaline and noradrenaline post-race respectively, despite being classified with a complete lesion and showing $\mathrm{OH}$ during the sit-up tilt test. Moreover, this was accompanied by an almost 4-fold increase in IL-6 following the race. Therefore, the impact of sympathetic reflexes and additional consequences of autonomic dysfunction other than lowered catecholamine concentrations on the acute inflammatory response to exercise would be an intriguing subject for future research.

While there is now sufficient evidence for the ability of upper-body exercise to induce an IL-6 response (e.g. Kouda et al., 2012; Paulson et al., 2013), this is not yet the case for eHsp72. In the current study no elevation of eHsp72 concentrations was detected in either CSCI or NON-CSCI. In a study investigating able-bodied participants, Leicht et al. (2016) reported an increase in eHsp72 after 45 min of arm-cranking at $60-65 \%$ peak power output in a non-permeable suit as opposed to the absence of an eHsp72 response after the same bout of exercise in conventional sport clothes. Since the exercise bout in the non-permeable suit 
resulted in increased heat storage, the results of this study suggest an important role of body temperature in the eHsp72 response. Since studies on lower-body exercise have shown increases in eHsp72 levels following relatively moderate bouts of exercise (Walsh et al., 2001), the reason for the attenuated eHsp72 response following upper-body exercise might partly lie in the limited muscle mass involved, inducing smaller increases in core temperature during exercise (Price, 2006). On the other hand, thermoregulation during exercise is impaired in individuals with SCI, possibly resulting in higher attained core temperatures (Price \& Campbell, 2003). Future research in applied settings should therefore attempt to monitor core temperature during competition to shed more light on this measure as a potential mediator of the inflammatory response (Laing et al., 2008; Whitham et al., 2007).

Some limitations of this study need consideration. First, we were not able to monitor sleep, warm-up practices, psychological stress prior and during the race, nor control food and liquid intake on the day of the race. These factors may have impacted on the acute inflammatory response following the race. Furthermore, the physiological underpinning of heart rate and blood pressure variability measures in the frequency domain have been the subject of debate (Billman, 2013). While it is indeed questionable whether LF HRV and LF SBP reflect solely sympathetic activation (Billman, 2013), the measures included in the present study strongly correlate with more clinical outcomes of autonomic dysfunction (Claydon \& Krassioukov, 2008). Moreover, these measures have been shown reliable in people with SCI (Ditor et al., 2005). Nevertheless, they should be considered as autonomic function indices, rather than measures of sympathetic activation (Esco et al., 2018). Additionally, the present study has assessed the relationship between autonomic function indices and the acute inflammatory response to strenuous exercise such as a wheelchair halfmarathon. Therefore, caution needs to be applied when translating the findings to shorter, 
moderate-intensity exercise prescribed to promote health in the general population with SCI

(Martin Ginis et al., 2018).

Notwithstanding its limitations, appreciating the scarcity of available data on the effects of exercise and autonomic dysfunction on health in people with SCI (Buker et al., 2018), the present field study in recreational wheelchair athletes may be used to inform future research in this area. For instance, the positive effect of chronic exercise training on autonomic balance (Buker et al., 2018) provides rationale for research into its impact on the acute inflammatory response to exercise, while further study is also needed to determine the most appropriate measures to indicate autonomic function in people with SCI (El-Kotob et al., 2017). Furthermore, there is increasing evidence for the anti-inflammatory effect of resistance training (Strasser et al., 2012). To further inform exercise prescription, future research could investigate the effectiveness of different types of (standardised) exercise in inducing an acute inflammatory response to people with SCI.

In summary, the strong association between post-race serum IL-6 and catecholamine concentrations suggests a major role for the latter in the acute inflammatory response to a wheelchair half-marathon. While autonomic function indices assessed at rest were not predictive of the IL- 6 response to the race when used in isolation, they enhanced the predictive value of autonomic function assessments when added to a predictive model with catecholamines alone. Therefore, the dampened acute IL-6 response to a wheelchair halfmarathon observed in people with CSCI may be influenced by more factors associated with autonomic dysfunction than solely blunted circulating catecholamine concentrations. Taking a wide range of factors associated with autonomic dysfunction into account may hence be of use to inform health promoting strategies to reduce chronic low-grade inflammation in individuals with CSCI. 
Disclosure of interest

1

2399

3

4

5400

6

7401

8

9

402

11

12403

13

14404

15

16

17

18

19406 20

21

The authors report no conflict of interest.

\section{Acknowledgements}

The authors are grateful for the financial support provided by the Peter Harrison

Centre and the Kyoten Research Center of Sports for Persons with Impairments. The authors also want to acknowledge Dr. Sonja de Groot (Reade Rehabilitation Centre, Amsterdam) and Dr. Carl Hulston (Loughborough University, Loughborough), who kindly provided the heart rate monitors used in this study. NCB acknowledges the support of the National Institute for Health Research (NIHR) Leicester Biomedical Research Centre. The views expressed are those of the authors and not necessarily those of the NHS, the NIHR or the Department of Health. 
Table 1. Characteristics of the participants with (CSCI) compared to the participants without (NON-CSCI) a cervical spinal cord injury. Values are given in mean (SD).

Table 2. Regression analyses with autonomic function indices and the inflammatory response to the wheelchair half-marathon. Reported as $\mathrm{R}^{2}(\mathrm{r})$

Fig. 1 Changes in circulating concentrations of IL-6, eHsp72, adrenaline and noradrenaline in response to the wheelchair half-marathon. Connected dots represent the individual responses, whilst horizontal lines represent the mean difference of the group between pre-and post-race, including the positive standard deviation of the difference. * Significant difference between pre- and post-race. ${ }^{\wedge}$ Significant time $\mathrm{x}$ group interaction between NON-CSCI and CSCI $(p<0.05)$. Graphs created using the template provided by Weissgerber et al. (Weissgerber, Milic, Winham, \& Garovic, 2015).

Fig. 2 Individual relationships between post-race concentrations of adrenaline, noradrenaline and eHsp72 with post-race IL-6 concentrations for CSCI (O) and NON-CSCI (+). * Variable significantly explains variance in post-race IL-6 concentrations $(p<0.05)$. 


\section{References}

Bauman, W. A., \& Spungen, A. M. (2008). Coronary heart disease in individuals with spinal cord injury: Assessment of risk factors. Spinal Cord, 46(7), 466-476.

Billman, G. E. (2013). The LF/HF ratio does not accurately measure cardiac sympatho-vagal balance. Frontiers in Physiology, 4, 1-5.

Bishop, N., Walsh, N., Haines, D., Richards, E., \& Gleeson, M. (2001). Pre-Exercise Carbohydrate Status and Immune Responses to Prolonged Cycling: II. Effect on Plasma Cytokine Concentration. International Journal of Sport Nutrition \& Exercise Metabolism, 11(4), 503-512

Buker, D. B., Oyarce, C. C., \& Plaza, R. S. (2018). Effects of spinal cord injury in heart rate variability after acute and chronic exercise: a systematic review. Topics in spinal cord injury rehabilitation, 24(2), 167-176.

Claydon, V. E., \& Krassioukov, A. V. (2008). Clinical correlates of frequency analyses of cardiovascular control after spinal cord injury. American Journal of Physiology. Heart and Circulatory Physiology, 294(2), 668-678.

Cohen J. (1992). A Power Primer. Psychological Bulletin, 112(1).

DeRijk, R. H., Boelen, A., Tilders, F. J., \& Berkenbosch, F. (1994). Induction of plasma interleukin-6 by circulating adrenaline in the rat. Psychoneuroendocrinology, 19(2), 155-163.

Ditor, D. S., Kamath, M. V., MacDonald, M. J., Bugaresti, J., McCartney, N., \& Hicks, A. L. (2005). Reproducibility of heart rate variability and blood pressure variability in individuals with spinal cord injury. Clinical Autonomic Research, 15(6), 387-393.

El-Kotob, R., Craven, B. C., Mathur, S., Ditor, D. S., Oh, P., Miyatani, M., \& Verrier, M. C. (2017). Assessing heart rate variability as a surrogate measure of cardiac autonomic function in chronic traumatic spinal cord injury. Topics in spinal cord injury rehabilitation, 24(1), 28-36.

Esco, M. R., Williford, H. N., Flatt, A. A., Freeborn, T., Nakamura, F. Y., \& Fedewa, M. V. (2018). Authors' reply to Medeiros et al.: Make it easier! Evaluation of the "vagal-sympathetic effect" in different conditions with R-R intervals monitoring. European Journal of Applied Physiology, 118(6), 1289-1290.

Fehrenbach, E., Niess, A. M., Voelker, K., Northoff, H., \& Mooren, F. C. (2005). Exercise intensity and duration affect blood soluble HSP72. International Journal of Sports Medicine, 26(7), 552557.

Fischer, C. P. (2006). Interleukin-6 in acute exercise and training: what is the biological relevance? Exerc Immunol Rev, 12(115), 6-33.

Freeman, R., Wieling, W., Axelrod, F. B., Benditt, D. G., Benarroch, E., Biaggioni, I., ... Van Dijk, J. G. (2011). Consensus statement on the definition of orthostatic hypotension, neurally mediated syncope and the postural tachycardia syndrome. Clinical Autonomic Research, 21(2), 69-72.

Jensen, J., Aslesen, R., Jebens, E., \& Skrondal, A. (1999). Adrenaline-mediated glycogen 
Johnson, J. D., \& Fleshner, M. (2006). Releasing signals, secretory pathways, and immune function of endogenous extracellular heat shock protein 72. Journal of Leukocyte Biology, 79(3), 425-434.

Krassioukov, A. (2009). Autonomic function following cervical spinal cord injury. Respiratory Physiology and Neurobiology, 169(2), 157-164.

Laing, S. J., Jackson, A. R., Walters, R., Lloyd-Jones, E., Whitham, M., Maassen, N., \& Walsh, N. P. (2008). Human blood neutrophil responses to prolonged exercise with and without a thermal clamp. Journal of Applied Physiology, 104(1), 20-26.

Langfort, J., Ploug, T., Ihlemann, J., Baranczuk, E., Donsmark, M., Górski, J., \& Galbo, H. (2003). Additivity of adrenaline and contractions on hormone-sensitive lipase, but not on glycogen phosphorylase, in rat muscle. Acta Physiologica Scandinavica, 178(1), 51-60.

Leicht, C. A., Goosey-Tolfrey, V. L., \& Bishop, N. C. (2013). Spinal cord injury: Known and possible influences on the immune response to exercise. Exercise Immunology Review, 19(0), 144-163.

Leicht, C. A., Kouda, K., Umemoto, Y., Banno, M., Kinoshita, T., Moriki, T., Tajima, F. (2015). Hot water immersion induces an acute cytokine response in cervical spinal cord injury. European Journal of Applied Physiology, 115(11), 2243-2252.

Leicht, C. A., Papanagopoulos, A., Haghighat, S., \& Faulkner, S. H. (2016). Increasing heat storage by wearing extra clothing during upper body exercise up-regulates heat shock protein 70 but does not modify the cytokine response. Journal of Sports Sciences, 35(17), 1752-1758.

Martin Ginis, K. A., Latimer, A. E., Arbour-Nicitopoulos, K. P., Buchholz, A. C., Bray, S. R., Craven, B. C., Wolfe, D. L. (2010). Leisure Time Physical Activity in a Population-Based Sample of People With Spinal Cord Injury Part I: Demographic and Injury-Related Correlates. Archives of Physical Medicine and Rehabilitation, 91(5), 722-728.

Martin Ginis, K. A., Van Der Scheer, J. W., Latimer-Cheung, A. E., Barrow, A., Bourne, C., Carruthers, P., Goosey-Tolfrey, V. L. (2018). Evidence-based scientific exercise guidelines for adults with spinal cord injury: An update and a new guideline. Spinal Cord, 56(4), 308-321.

Midi, H., Sarkar, S. K., \& Rana, S. (2010). Collinearity diagnostics of binary logistic regression model. Journal of Interdisciplinary Mathematics, 13(3), 253-267.

Ogawa, T., Nakamura, T., Banno, M., Sasaki, Y., Umemoto, Y., Kouda, K., Tajima, F. (2014). Elevation of interleukin- 6 and attenuation of tumor necrosis factor- $\alpha$ during wheelchair half marathon in athletes with cervical spinal cord injuries. Spinal Cord, 52(8), 601-605.

Paulson, T. A. W., Goosey-Tolfrey, V. L., Lenton, J. P., Leicht, C. A., \& Bishop, N. C. (2013). Spinal cord injury level and the circulating cytokine response to strenuous exercise. Medicine and Science in Sports and Exercise, 45(9), 1649-1655.

Petersen, A. M. W. \& Pedersen, B. K. (2005). The anti-inflammatory effect of exercise. J Appl Physiol, 98, 1154-1162. 
Price, M. J. (2006). Thermoregulation during Exercise in Individuals with Spinal Cord Injuries. Sports Med, 36(10), 863-879.

Price, M. J., \& Campbell, I. G. (2003). Effects of spinal cord lesion level upon thermoregulation during exercise in the heat. Medicine and Science in Sports and Exercise, 35(7), 1100-1107.

Sasaki, Y., Furusawa, K., Tajima, F., Nakamura, T., Kouda, K., Kanno, N., Shimizu, K. (2014). Wheelchair marathon creates a systemic anti-inflammatory environment in persons with spinal cord injury. Clinical Journal of Sport Medicine, 24(4), 295-301.

Scheer, J. W. Van Der, Christof, Y. K., Philip, A. L., Manabu, J. M., \& Tajima, V. L. G. F. (2018). A comparison of static and dynamic cerebral autoregulation during mild whole-body cold stress in individuals with and without cervical spinal cord injury: a pilot study. Spinal Cord, 56(5), 469477.

Steensberg, A., Van Hall, G., Osada, T., Sacchetti, M., Saltin, B., \& Klarlund Pedersen, B. (2000). Production of interleukin- 6 in contracting human skeletal muscles can account for the exerciseinduced increase in plasma interleukin-6. J Physiol, 529(1), 237-242.

Steensberg, A., Toft, A D., Schjerling, P., Halkjaer-Kristensen, J., \& Pedersen, B. K. (2001). Plasma interleukin-6 during strenuous exercise: role of epinephrine. American Journal of Physiology. Cell Physiology, 281(3), 1001-1004.

Strasser, B., Arvandi, M., \& Siebert, U. (2012). Resistance training, visceral obesity and inflammatory response: A review of the evidence. Obesity Reviews, 13(7), 578-591.

Umemoto, Y., Furusawa, K., Kouda, K., Sasaki, Y., Kanno, N., Kojima, D., \& Tajima, F. (2011). Plasma IL-6 levels during arm exercise in persons with spinal cord injury. Spinal Cord, 49(12), $1182-1187$.

Walsh, R. C., Koukoulas, I., Garnham, A., Moseley, P. L., Hargreaves, M., \& Febbraio, M. A. (2001). Exercise increases serum Hsp72 in humans. Cell Stress and Chaperones, 6(4), 386-393.

Weissgerber, T. L., Milic, N. M., Winham, S. J., \& Garovic, V. D. (2015). Beyond Bar and Line Graphs: Time for a New Data Presentation Paradigm. PLoS Biology, 13(4), 1-10.

West, C. R., Gee, C. M., Voss, C., Hubli, M., Currie, K. D., Schmid, J., \& Krassioukov, A. V. (2015). Cardiovascular control, autonomic function, and elite endurance performance in spinal cord injury. Scandinavian Journal of Medicine \& Science in Sports, 25(4), 476-485.

Whitham, M., Laing, S. J., Jackson, A., Maassen, N., \& Walsh, N. P. (2007). Effect of exercise with and without a thermal clamp on the plasma heat shock protein 72 response. Journal of Applied Physiology, 103(4), 1251-1256.

Zhang, Y., Lin, J. X., \& Vilcek, J. (1988). Synthesis of interleukin 6 (interferon-beta 2/B cell stimulatory factor 2) in human fibroblasts is triggered by an increase in intracellular cyclic AMP. The Journal of Biological Chemistry, 263(13), 6177-6182. 
1 2568

3

4

5

9

10

11

12

13

14

15

16

17

18

19

20

21

22

23

24

25

26

27

28

29

30

31

32

33

34

35

36

37

38

39

40

41

42

43

44

45

46

47

48

49

50

51

52

53

54

55

56

57

58

59

60

61

62

63

64

65 
Table 1. Characteristics of the participants with (CSCI) compared to the participants without (NON-CSCI) a cervical spinal cord injury. Values are given in mean (SD).
Parameter
NON-CSCI $(\mathrm{N}=10)$

\section{CSCI $(\mathrm{N}=7)$}

NON-SCI vs. CSCI

mean $(95 \% \mathrm{CI})$

difference

\begin{tabular}{|c|c|c|c|}
\hline Age (yrs) & $43.5(12.1)$ & $43.4(15.0)$ & $-0.7(-14.1$ to 13.9$)$ \\
\hline Height $(\mathrm{cm})$ & $155.3(23.3)$ & $172.6(7.8)$ & $17.3(-2.4$ to 37.0$)$ \\
\hline Body mass $(\mathrm{kg})$ & $53.8(11.4)$ & $60.0(15.8)$ & $6.2(-8.4$ to 20.8$)$ \\
\hline Wheelchair racing experience (yrs) & $15.1(9.6)$ & $16.3(10.2)$ & $1.2(-11.0$ to 13.4$)$ \\
\hline Training per week (min) & $313(245)$ & 307 (193) & $-5(-273$ to 262$)$ \\
\hline Lesion level SCI & $<$ Thoracic 5 & $>$ Cervical 8 & NA \\
\hline $\begin{array}{l}\text { Sensory and motor complete/incomplete } \\
\text { SCI }\end{array}$ & $\mathrm{N}=4 / 1$ & $\mathrm{~N}=3 / 4$ & $-1 / 3$ \\
\hline Orthostatic hypotension & $\mathrm{N}=1$ & $\mathrm{~N}=4$ & 3 \\
\hline Change in SBP/DBP following tilt ( $\mathrm{mmHg}$ ) & $-2(12) /-4(7)$ & $-21(10) /-5(11)$ & $\begin{array}{c}-18.1(-32.3 \text { to }-3.7)^{*} / \\
-0.9(-11.0 \text { to } 9.2)\end{array}$ \\
\hline Supine LF/HF RRI & $1.65(1.22)$ & $0.34(0.20)$ & $-1.31(-2.53 \text { to }-0.36)^{*}$ \\
\hline Supine LF SBP $(\mathrm{Hz})$ & $7.54(3.71)$ & $1.39(1.95)$ & $-6.15(-10.07 \text { to }-2.22)^{*}$ \\
\hline Supine TP SBP (Hz) & $5.96(3.74)$ & $36.34(11.27)$ & $-30.3(-41.9 \text { to }-18.9)^{*}$ \\
\hline Supine RMSSD (ms) & $42.6(11.1)$ & $46.6(23.1)$ & $3.95(-46.0$ to 53.9$)$ \\
\hline
\end{tabular}

Abbreviations: $\mathrm{SBP}=$ systolic blood pressure; $\mathrm{DBP}=$ diastolic blood pressure; $\mathrm{HR}=$ heart rate; $\mathrm{LF} / \mathrm{HF} \mathrm{RRI}=$ ratio of the power in the low and high frequency domain of the heart rate variability; LF SBP = power in the low frequency domain of systolic blood pressure variability; TP SBP = total power of systolic blood pressure variability. Orthostatic hypotension is defined as a drop in $\mathrm{SBP}>20 \mathrm{mmHg}$ or $\mathrm{DBP}>10 \mathrm{mmHg}$ following the onset of the sitting position. * Significant difference between NON-CSCI and CSCI $(p<0.05)$. 
Table 2. Regression analyses with autonomic function indices and the inflammatory response to the wheelchair half-marathon. Reported as $\mathrm{R}^{2}(\mathrm{r})$

Predictor variable IL-6 post-race Adrenaline post-race $\quad$ Noradrenaline post-race

\begin{tabular}{|c|c|c|c|}
\hline A & $44 \%(0.66)^{*}$ & N/A & $67 \%(0.82)^{*}$ \\
\hline $\mathrm{N}$ & $8 \%(0.29)$ & $67 \%(0.82)^{*}$ & N/A \\
\hline LF/HF RRI & $8 \%(0.28)$ & $29 \%(0.54)^{*}$ & $11 \%(0.33)$ \\
\hline LF SBP & $12 \%(0.35)$ & $19 \%(0.44)$ & $34 \%(0.58)^{*}$ \\
\hline TP SBP & $29 \%(0.54)$ & $33 \%(0.58) *$ & $28 \%(0.53)$ \\
\hline RMSSD & $11 \%(0.33)$ & $5 \%(0.23)$ & $3 \%(0.18)$ \\
\hline $\mathrm{A}+\mathrm{N}$ & $60 \%(0.77)^{*}$ & N/A & N/A \\
\hline LF/HF RRI + LF SBP & $14 \%(0.36)$ & $33 \%(0.58)$ & $34 \%(0.59)$ \\
\hline $\mathrm{LF} / \mathrm{HF}$ RRI + TP SBP & $27 \%(0.52)$ & $38 \%(0.61)$ & $25 \%(0.50)$ \\
\hline RMSSD + LF SBP & $6 \%(0.24)$ & $21 \%(0.46)$ & $34 \%(0.58)$ \\
\hline RMSSD + TP SBP & $34 \%(0.58)$ & $36 \%(0.60)$ & $30 \%(0.55)$ \\
\hline $\mathrm{A}+\mathrm{N}+\mathrm{LF} / \mathrm{HF}$ RRI + LF SBP & $81 \%(0.90)^{*}$ & N/A & N/A \\
\hline $\mathrm{A}+\mathrm{N}+\mathrm{LF} / \mathrm{HF} \mathrm{RRI}+\mathrm{TP} \mathrm{SBP}$ & $88 \%(0.94)^{*}$ & N/A & N/A \\
\hline $\mathrm{A}+\mathrm{N}+\mathrm{RMSSD}+\mathrm{LF} \mathrm{SBP}$ & $64 \%(0.80)$ & N/A & N/A \\
\hline $\mathrm{A}+\mathrm{N}+\mathrm{RMSSD}+\mathrm{TP}$ SBP & $72 \%(0.85)^{*}$ & N/A & N/A \\
\hline
\end{tabular}

Abbreviations: IL-6 = interleukin-6; A = adrenaline post-race; $\mathrm{N}=$ noradrenaline post-race; LF/HF RRI = ratio of the power in low and high frequency of the heart rate variability; LF SBP = power in the low frequency of systolic blood pressure variability; TP SBP = total power of systolic blood pressure variability; RMSSD = root mean square differences of successive R-R intervals.

* Regression model significantly explains variance in dependent variable $(p<0.05)$ 

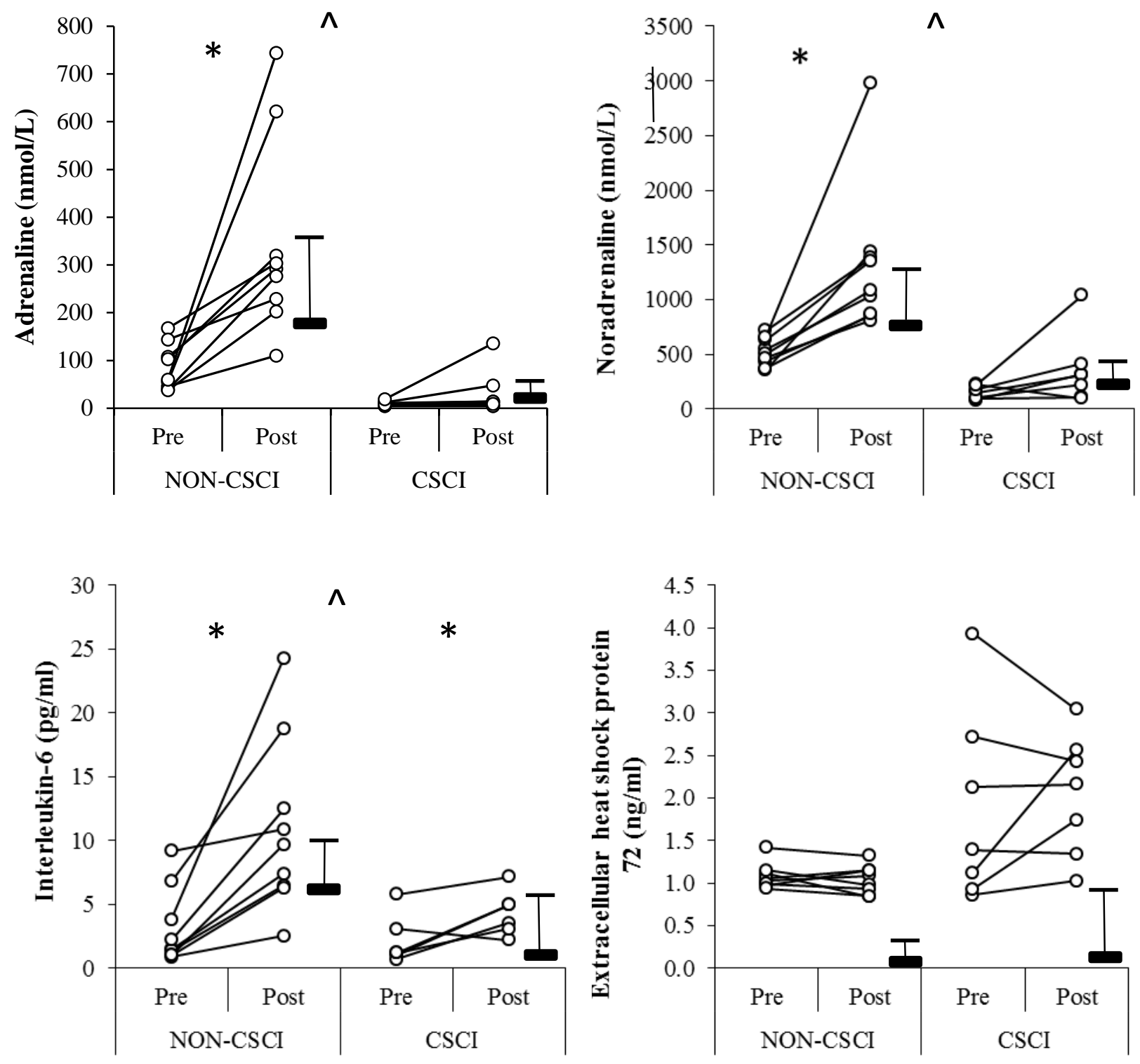

Fig. 1 Changes in serum concentrations of IL-6, eHsp72, adrenaline and noradrenaline in response to the wheelchair half-marathon. Connected dots represent the individual responses, whilst horizontal lines represent the mean difference of the group between pre-and post-race, including the positive standard deviation of the difference. * Significant difference between pre- and post-race. ${ }^{\wedge}$ Significant time $\mathrm{x}$ group interaction between NON-CSCI and CSCI $(p<0.05)$. Graphs created using the template provided by Weissgerber et al. (Weissgerber, Milic, Winham, \& Garovic, 2015). 


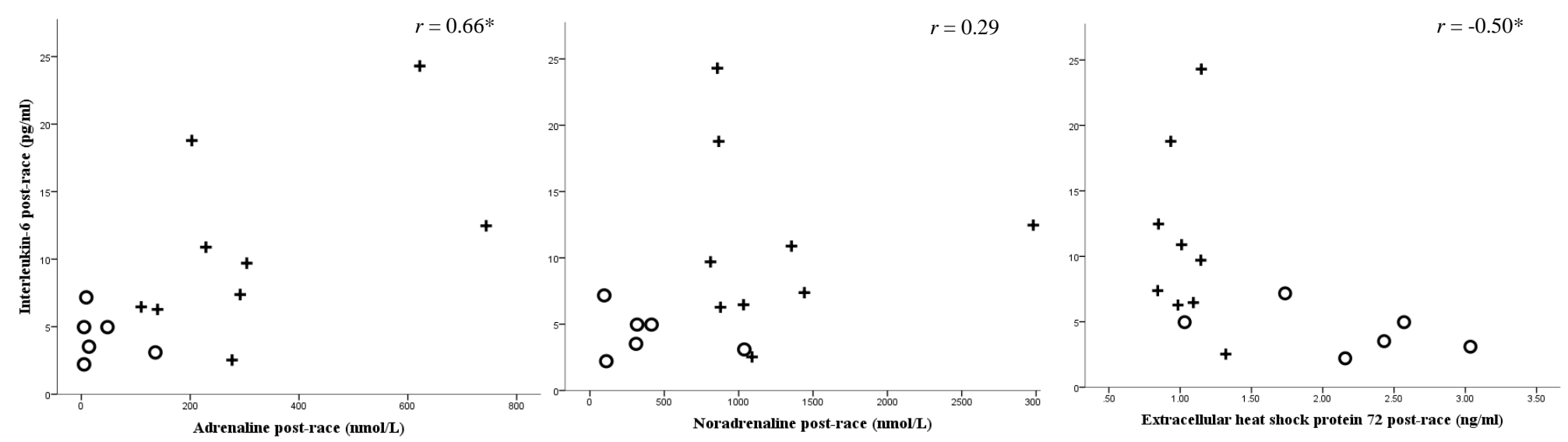

Fig. 2 Individual relationships between post-race serum concentrations of adrenaline, noradrenaline and eHsp72 with post-race IL-6 serum concentrations for CSCI (O) and NON-CSCI (+). * Variable significantly explains variance in post-race IL-6 serum levels $(p<0.05)$. 